DOI:10.2174/2211352518999200525143254

\title{
Nanocluster structure of pistacia atlantica subsp. Kurdica turpentine and its antibacterial effects
}

Darehbagh R.R. ${ }^{\mathrm{a}, \mathrm{b}, \mathrm{c}}$,Ramezani R. ${ }^{\mathrm{a}}$, Hosseinpanahi A. ${ }^{\mathrm{d}}$, Fotoohi A. ${ }^{\mathrm{a}}$, Rouhi S.*e

${ }^{a}$ Student Research Committee, Kurdistan University of Medical Sciences, Sanandaj, Iran

${ }^{\mathrm{b}}$ Kurdistan Gum Manufactur-ing Company (VAN), Sanandaj, Iran

${ }^{\mathrm{c}}$ Nano Experts Organization (NEO), Iran

${ }^{\mathrm{d}}$ Department of Microbiology, Faculty of Medicine, Kurdistan University of Medical Sciences, Sanandaj, Iran

${ }^{\mathrm{e}}$ Medical Microbiology Research Center, Qazvin University of Medical Sciences, Qazvin, Iran

Background: Medicinal herbs such as Pistacia Atlantica (P. Atlantica) subsp. Kurdica have antimicrobial effects. The present study is aimed to investigate the nanocluster structure of $\mathrm{P}$. atlantica subsp. Kurdica turpentine and its composing elements and antibacterial effect. Methods: $100 \mu \mathrm{l}$ ethanol was used to dissolve oily turpentine. $2,2.2,2.4$, and $2.6 \mu \mathrm{g} / \mu \mathrm{l}$ of turpentine were used for investigating the antibacterial effects using disk and well diffusion methods. Elemental and nanocluster structure analyses were performed by Energy-Dispersive X-Ray Microa-nalysis (EDXMA) and Field Emission (FE)-scanning electron. Two-way analysis of variance (ANOVA) and Bonferroni test were used for data analysis $(\mathrm{p} \leq 0.001)$. Results and Discussion: EDXMA elemental analysis of turpentine included: Zinc (Zn), Magnesi-um (Mg), Fluorine (F), Oxygen (O), Silicon (Si), Carbon (C), and Argentum (Ag). A topography image of the turpentine showed a nanocluster surface with bright clusters in the background. The largest diameters of the growth inhibition zones $(24.67 \pm 0.58 \mathrm{~mm}$ in the disk diffusion and $23.67 \pm 1.53 \mathrm{~mm}$ in the well diffusion) that were created by turpentine were observed against S. aureus ATCC 25923 at the concentration of $2.6 \mu \mathrm{g} / \mu \mathrm{l}$. The diameter of the inhibition zone around bacterial growth had a direct relationship with turpentine concentration $(\mathrm{p} \leq 0.001)$. Conclusion: The nanocluster structure of turpentine and its composed elements were detected in this research. Moreover, the antibacterial effects of turpentine were proved. Herbal substances are widely used in medical applications. Different elements of P. atlantica subsp. Kurdica turpentine can be used as antibacterial agents, but more invitro and in-vivo studies should be performed in this field. 